\title{
Correction: ZNF774 is a potent suppressor of hepatocarcinogenesis through dampening the NOTCH2 signaling
}

\author{
Chengjian Guan · Lin He · Zhenyu Chang $\cdot$ Xinjin Gu • Jing Liang $(\mathbb{D} \cdot$ Rong Liu
}

Published online: 3 February 2020

(c) The Author(s), under exclusive licence to Springer Nature Limited 2020

\section{Correction to: Oncogene}

https://doi.org/10.1038/s41388-019-1075-0

The original version of this Article omitted the following from the Acknowledgements:

This paper was supported by Capital Health Research and Special Development (No. 2016-1-1111), National Key
Technologies R\&D Program (No. 2015BAI13B09), Beijing Municipal Administration of Hospitals Incubator Program (Nos. PX2016001 and PX2019004) and "Miaopu" Innovation Foundation of the Chinese PLA General Hospital (No. 17KMM07).

This has now been corrected in both the PDF and HTML versions of the Article. 